\title{
Small bowel obstruction due to subserosal endometriosis: an elusive condition
}

\author{
Garima Mishra ${ }^{1}$, Manu Vats ${ }^{2}$, Diwakar Pandey ${ }^{2}$, Azaz Akhtar ${ }^{2}$
}

\begin{abstract}
${ }^{1}$ Department of Obstetrics and Gynaecology, Mahatma Gandhi Memorial Medical College, Indore, MP, India
${ }^{2}$ Department of General Surgery, Lady Hardinge Medical College, New Delhi, India
\end{abstract}

Received: 01 January 2015

Revised: 02 January 2015

Accepted: 08 February 2016

\author{
*Correspondence: \\ Dr. Diwakar Pandey, \\ E-mail: drdiwakarpandey86@gmail.com
}

Copyright: (c) the author(s), publisher and licensee Medip Academy. This is an open-access article distributed under the terms of the Creative Commons Attribution Non-Commercial License, which permits unrestricted non-commercial use, distribution, and reproduction in any medium, provided the original work is properly cited.

\begin{abstract}
The bowel is involved in 3.8 to $37 \%$ of women with endometriosis, out of which nearly $1 \%$ present with signs of bowel obstruction to the surgeon. This report describes a case of acute small bowel obstruction in a 23 years old woman. The patient gave a history of intermittent episodes of pain abdomen and abdominal distension for the past 1 year with significant loss of weight. The menstrual cycles were normal. Although there was no history of Tuberculosis, a positive history of contact was present. The clinical and biochemical picture was suggestive of peritonitis. CECT of the abdomen revealed a long segment distal ileal stricture. With a provisional diagnosis of Tubercular Ileo-Caecal stricture perforation, a midline exploratory laparotomy was performed. The procedure consisted of right limited hemicolectomy and primary ileo-ascending anastomosis with a proximal loop ileostomy. Ileostomy was done to allow the healing of distal anastomosis and closure was done after 4 weeks. Histopathology of the resected segment of ileum revealed subserosal endometriosis. Postoperatively, the patient was not given any hormonal therapy and recovery has been uneventful over the past 1 year of follow up.
\end{abstract}

Keywords: Endometriosis, Small bowel, Small bowel obstruction

\section{INTRODUCTION}

Endometriosis of the small bowel is a rare disease, with only $7 \%$ of women with endometriosis having small bowel involvement. ${ }^{1}$ The presentation is often enigmatic, ranging from asymptomatic to a constellation of symptoms, like abdominal pain, constipation or diarrhoea, rectal pain, infertility, abdominal mass, increased urinary frequency and acute small bowel obstruction. Acute small bowel obstruction is reported in around $0.7 \%$ of the patients with endometriosis who undergo surgical intervention., ${ }^{2,3}$ Preoperatively, the patient is often misdiagnosed with a common cause of small bowel obstruction viz. adhesive obstruction, Tubercular stricture obstruction, Crohn's disease or malignancy. Therefore, most of times definite diagnosis is obtained postoperatively in the histopathology report. The authors report a case of 23 years old woman who presented with small bowel obstruction and was provisionally diagnosed with tubercular stricture perforation of ileo-caecal region. She was treated with right limited hemicolectomy with primary ileo-ascending anastomosis and proximal loop ileostomy. The histopathology report of the resected specimen contradicted our preoperative diagnosis and was suggestive of subserosal endometriosis. Follow up is uneventful for past 1 year.

\section{CASE REPORT}

A 23 years old $\mathrm{P}_{2} \mathrm{~L}_{2}$ woman presented with the complaints of abdominal pain, bilious vomiting and 
abdominal distension since 2 days. This was associated with diarrhoea and low grade fever. There was no history of any altered bowel habits, melena or hematochezia. Patient had a history of similar episodes, thrice in the past one year. She was managed conservatively each time by nasogastric tube decompression, analgesics and antibiotics at primary health care centres. Upon discharge from the health centre, she was advised to consult and undergo detailed evaluation at a higher tertiary centre, which she ignored. There was a significant gradual weight loss since last one year and she also gave a history of contact with a pulmonary tuberculosis patient. She had undergone a lower segment caesarean section for obstructed labour 2 years back. Personal, Family and Menstrual history were essentially unremarkable. On physical examination, dehydration and fever were present. On inspection, abdominal distension was present. Generalised abdominal tenderness and guarding was appreciated on palpation and bowel sounds were sluggish on auscultation. No lymphadenopathy or organomegaly was present. Digital rectal examination and proctoscopy were normal. A provisional diagnosis of tubercular perforation peritonitis was reached upon, based on the typical history and physical examination.

Haematological investigations, at the time of admission, revealed leucococytosis $\left(15600 / \mathrm{cm}^{3}\right)$ with neutrophilia $(81 \%)$, Haemoglobin of $14 \mathrm{~g} / \mathrm{dl}$, Platelets $1.68 \mathrm{lakh} / \mathrm{cm}^{3}$, CRP $12 \mathrm{mg} / \mathrm{L}$, Urea $4.7 \mathrm{mmol} / \mathrm{L}$, Serum Creatinine 41 micro moles/L, Serum Albumin 5.1 g/dl with normal Liver enzymes.

Chest roentgenogram was unremarkable with absence of any old calcified lesion (suggestive of TB), pleural nodule or thickening. No free gas under the domes of diaphragm was visualized. Erect X-ray of the abdomen showed dilated small bowel loops with multiple air fluid levels (Figure 1 and 2).

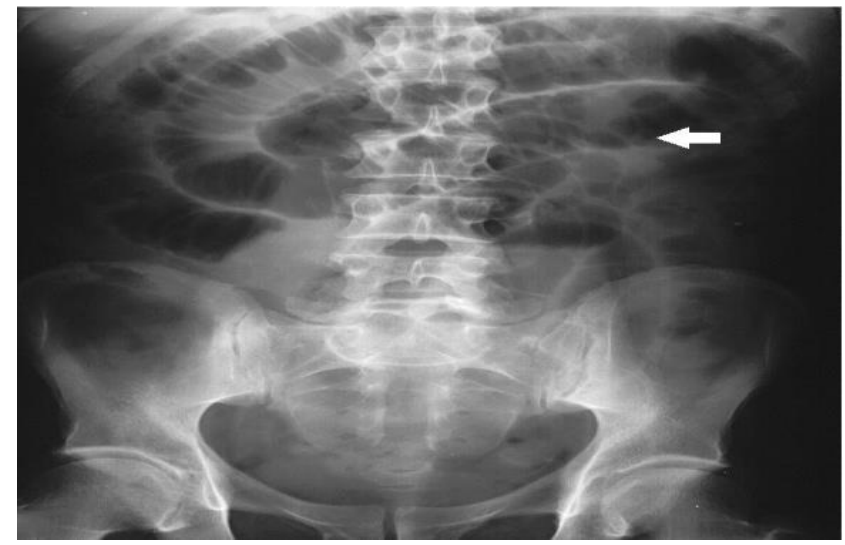

Figure 1: Supine X Ray Abdomen showing dilated gas filled loops of small Intestine.

CECT scan of the abdomen was suggestive of dilated jejunal and proximal ileal loops (up to 3.5 to $4.2 \mathrm{~cm}$ in diameter) with fluids levels. A long segment narrowing and thickening (maximum thickness $8 \mathrm{~mm}$ ) of distal ileal loop, likely to be a stricture, was visible. There was evidence of multiple mesenteric lymphadenopathy and mild free fluid in pelvis. Presence of simple cortical cysts in both the kidneys was observed. The rest of the abdominal organs, uterus and bilateral adenexa were normal and there was no retroperitonal lymphadenopathy (Figure 3 and 4).

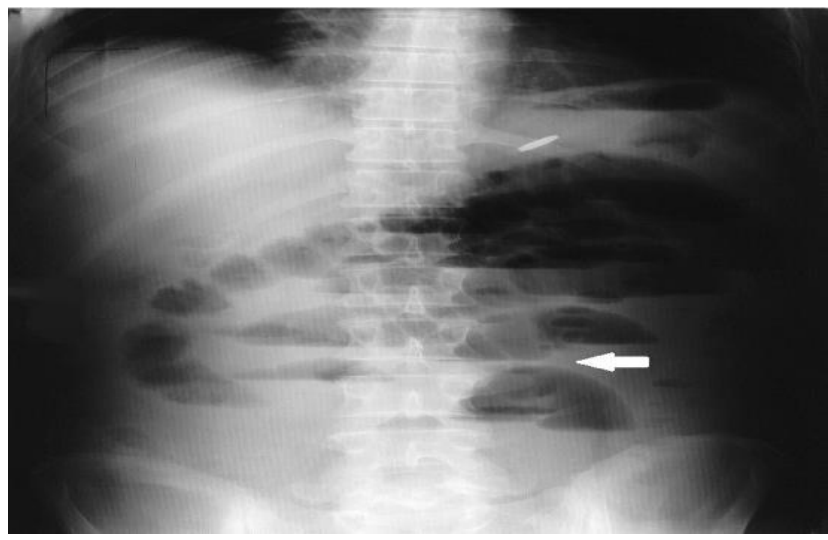

Figure 2: Erect X Ray Abdomen showing multiple air fluid levels in small bowel.

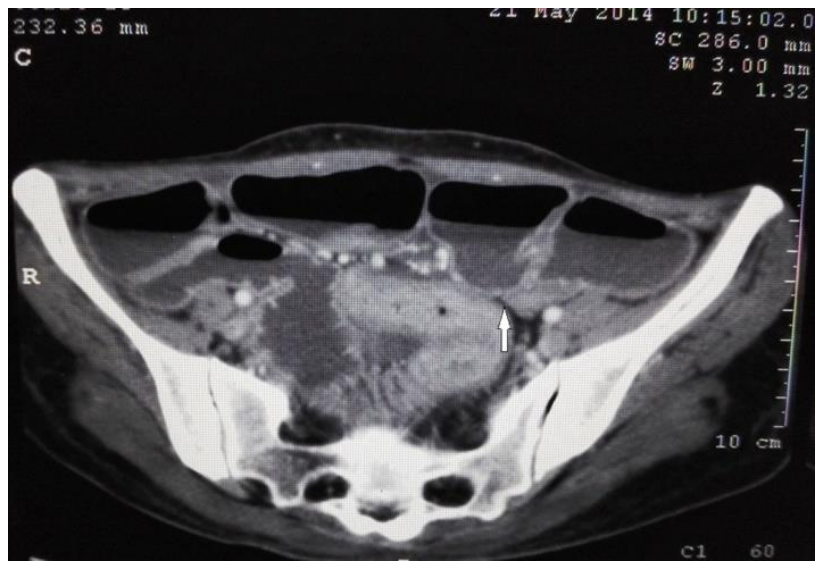

Figure 3: Contrast enhanced CT abdomen (axial view) suggestive of Ileal stricture (transition point shown in arrow) with proximal dilated small bowel and distal collapsed bowel loops.

With a positive history of contact with pulmonary tuberculosis, weight loss, multiple episodes of sub acute intestinal obstruction; CECT finding suggestive of ileal thickening and mesenteric lymphadenopathy; high prevalence of tuberculosis in India, our first differential diagnosis was tubercular ileal stricture with perforation peritonitis.

However, apart from this, the following additional differential diagnosis can be kept in mind in the Indian subcontinent with decreasing order of relevance:

1. Worm infestation (Ascaris lumbricoides, strongyloides stercolis) with small bowel obstruction. 
2. Infective diseases like Enteric fever, Yersinia enterocolitis.

3. Diverliculitis.

4. Inflammatory bowel disease.

5. Malignancy (Carcinoid tumor, Lymphoma).

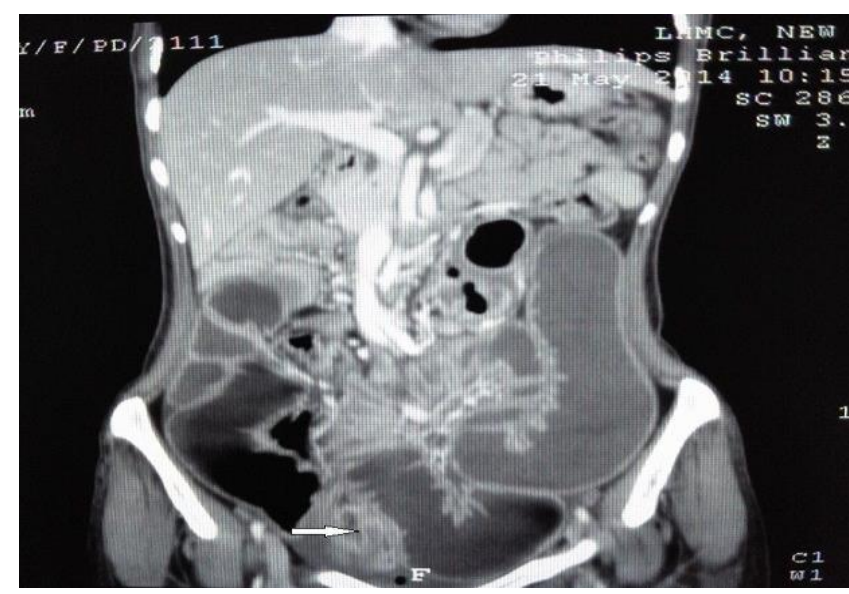

Figure 4: Contrast enhanced CT abdomen (coronal section) shows distended fluid filled small bowel loops. The transition point appears as circumferential mural thickening with hypoattenuated outer layer (arrow).

After adequate resuscitation and evaluation, patient was shifted for emergency surgery under general anaesthesia. The surgical procedure included an abdominal exploration through a midline laparotomy. There was minimal serous free fluid and no evidence of appendicular perforation. On further exploration, a distal ileal stricture extending to the ileo-caecal junction was identified. The proximal ileum and jejunum were dilated but looked grossly healthy. Multiple subcentimetric mesenteric lymph nodes were present. Colon, uterus, bilateral ovaries and fallopian tubes appeared normal. Since the enlarged mesenteric lymph nodes were predominantly present around the ileo-caecal junction alongwith the stricture; we decided to proceed with a right limited hemicolectomy with primary ileo-ascending anastomosis. A proximal loop ileostomy was formed to allow the healing of the distal anastomosis. A pelvic drain was placed in situ followed by closure of abdomen in layers.

Post-operative recovery was uneventful. Patient was gradually allowed oral feeds starting on $3^{\text {rd }}$ post operative day. Pelvic drain was removed on post-operative day 7 .

Histopathological examination report of the resected specimen was suggestive of dilated segments of ileum with increased bowel thickness and presence of nests of endometrial glands and stroma in muscularis propria (Figure 5). No pathological abnormalities were detected in resected caecum and a part of ascending colon. Mesenteric lymph nodes showed reactive hyperplasia with no evidence of endometrial glands. Patient was referred to the Department of Obstetrics and Gynaecology at her home town. Patient was not offered any hormonal therapy postoperatively and she has remained symptom free and gained some weight over the past 1 year of follow up.

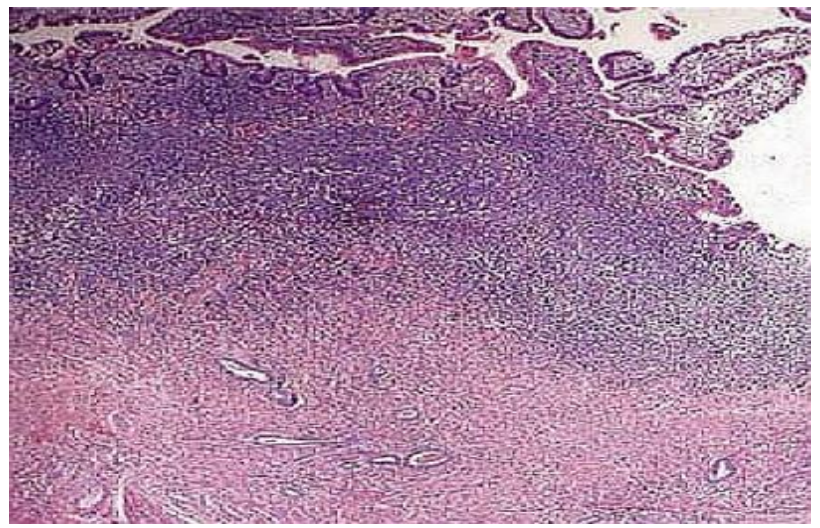

Figure 5: Histopathology (H\&E 40X) of resected ileal wall. Glandular structure having endometrial epithelial lining is infiltrating the submucosal layer of ileum with lymphoid hyperplasia in mucosa and lamina propria.

\section{DISCUSSION}

Endometriosis, first described by Von rokitansky in 1860 , is defined as the presence of benign endometrial tissue outside the uterus. The pelvic organs and pelvic peritoneum is the most common site of endometriosis. ${ }^{4}$ Outside the pelvic cavity, it can be found in intestines, abdominal wall (particularly near scar site of previous caesarean section) and as distant as lungs, skin and the central nervous system. ${ }^{5}$ It has been reported that caesarean section is associated with $66.7 \%$ of cases of abdomino-pelvic endometriosis. ${ }^{6}$

Bowel endometriosis occurs in between 3.8 to $37 \%$ of women with endometriosis. ${ }^{7,8}$ Affected sites in intestine in decreasing order of frequency are recto-sigmoid (72\%), rectovaginal septum (13\%), small bowel (7\%), caecum (4\%) and appendix (3\%). ${ }^{1}$ Involvement of small bowel proximal to the Meckel's diverticulum is rare, with only a few reported cases found in literature. ${ }^{9,10}$

The aetiology of endometriosis is still elusive .Various theories have been propounded to explain the migration of endometrial tissues to ectopic sites: ${ }^{11-14}$

1. Reflux of Endometrial tissues from fallopian tube during menstrual cycle (Sampsons retrograde menstruation theory) leading to implantation of these tissues into abdominal organs. ${ }^{11,13}$

2. Metaplastic changes in pluripotent peritoneal mesothelium with lymphatic or hematogenous migration (Minh's theory). ${ }^{11}$

3. Metaplastic changes in mullerian remnants leading to formation of endometrial nodule (Donnez et all). ${ }^{15}$ 
4. Neurologic hypothesis which advocates that infiltration of bowel wall by endometrial tissues occurs along the nerves at a distance from primary lesion. $^{16}$

Based on the location in the gastro-intestinal tract they can further be classified into superficial/ serosal endometriosis, intestinal/ subserosal endometriosis and endometrial mass.

The symptomatology revolves around the mass effect of endometriosis leading to obstructive symptoms with cyclical trends corresponding with menstruation or due to release of inflammatory and angiogenic cytokines in the surrounding tissues leading to localised fibrosis and stricture formation. ${ }^{17,18}$ The resulting fibrosis and stricture formation may be the reason behind most intestinal endometriosis lacking the classical cyclical abdominal pain during menstrual cycle.

Bowel endometriosis is usually not encountered first by a gynaecologist, but by a general surgeon or gastroenterologist due to its vague symptom complex of abdominal pain, distension, cramps, diarrhoea, increased urinary frequency or cyclical fresh rectal bleed. ${ }^{2}$ The true incidence of bowel obstruction caused by endometriosis is not known but it is reported in less than $1 \%$ of the intestinal endometriosis cases, usually affecting the rectosigmoid colon. ${ }^{3}$ Small bowel obstruction has been observed in only $0.7 \%$ of all the surgical interventions carried out for endometriosis. ${ }^{3}$

Small bowel obstruction caused by Endometriosis is usually diagnosed postoperatively by the histopathology report. Endometriosis is often confused with Tubercular enteritis, Crohn's ileitis, Yersinia enterocolitis and malignancy. Crohn's ileitis resembles bowel endometriosis maximally, as both are having a hallmark feature of patchy involvement of intestines with intervening normal uninvolved skip areas. Intraoperatively, this causes a great diagnostic dilemma.

There is no gold standard investigation that is recommended to diagnose intestinal endometriosis. However, MRI is one of the techniques most commonly used. MRI has a sensitivity of $88 \%$, specificity of $98 \%$, positive predictive value of $95 \%$ and a negative predictive value of $95 \%$ and an accuracy of $95 \%$ in diagnosing intestinal endometriosis. ${ }^{19}$

Small hemorrhagic endometrial nodules are difficult to be picked up by MRI, owing to their similar signal intensity with surrounding muscular structure. Loubeyre $\mathrm{P}$ et al have reported a novel technique of inserting ultrasonography jelly into the vagina and rectum during MRI to enhance the identification of small endometrial intestinal nodules. ${ }^{20}$

Multi detector computerized tomography enteroclysis (MDCT-e) has recently been found to be having great potential in diagnosing intestinal endometriosis. In a study by Biscaldi et al, out of a total of 98 patients with symptoms of colorectal endometriosis, MDCT-e detected $94.8 \%$ of endometrial nodules. ${ }^{21}$

Transvaginal ultrasonography has been traditionally used for colorectal endometriosis but the probability of accurately identifying the lesions depends upon the experience and acumen of the operator. Similarly, the role of colonoscopy in the diagnosis of endometriosis is limited, because mucosal invasion in intestinal endometriosis is seen in only $10 \%$ of cases. ${ }^{22,23}$

Recently, a keen interest in the use of serum markers, like CA125, to monitor the progression of endometriosis has developed. ${ }^{24}$ Other markers which have been evaluated are CA 19.9 and cytokine Interleukin-6 (IL-6). Cytokine Interleukin 6 (IL-6) has been found to be more sensitive and specific than CA 125 , however no serum marker till now has been proven to be accurate enough for use in clinical practice. ${ }^{25}$

Surgery is the preferred modality of treatment for intestinal endometriosis. Nodulectomy or segmental bowel resection are usually done. However, progestins and gonadotrophin releasing hormone analogues may be offered in patients of symptomatic colonic endometriosis, with less than $60 \%$ stenosis of bowel lumen, who wish to defer surgery and do not wish to conceive. ${ }^{26,27}$ Hormonal therapy, however, has a disadvantage of frequent relapse and progression of endometrial lesions, which almost always requires surgery at a later stage of disease. A few of the unfit patients with gross pelvic and rectosigmoid endometriosis and those who are awaiting surgery have been treated temporarily with colonic stenting. ${ }^{28}$ In small bowel endometriosis, the treatment of choice remains surgery and there is no described role of hormonal therapy. According to a recent meta-analysis, postoperative hormonal therapy does not demonstrate any benefits. $^{29}$

Microscopically, endometriotic foci in the bowel wall are usually composed of aggregates of small, often widely spaced endometrioid glands embedded within a variable amount of endometrial stroma. ${ }^{14}$ Immunohistochemistry reveals CD10 positivity, which is a strong marker of endometriosis and can be used to make an accurate diagnosis. ${ }^{30}$

Bowel endometriosis may present with a multitude of signs and symptoms and thus create a diagnostic dilemma in the surgeon's mind. However, it should always be kept as a differential diagnosis of bowel obstruction in a woman of reproductive age group. Owing to the complexities of reaching to an accurate and prompt diagnosis and thus initiating appropriate management of the patient, a multidisciplinary team comprising of the General surgeon, Gynaecologist and Gastroenterologist is indispensable. 


\section{CONCLUSIONS}

1. Endometriosis is a rare cause of intestinal obstruction, which requires a high index of suspicion, especially in women of reproductive age group with a history of caesarean section.

2. Multidisciplinary care should be encouraged to ensure correct evaluation and management.

3. Surgical intervention is the mainstay of treatment for bowel endometriosis with no role of post-operative hormone therapy.

4. Recent interest has been developed to evaluate CA 125, IL-6 and CA 19.9 as markers to monitor progression of disease, albeit with discouraging results.

Funding: No funding sources

Conflict of interest: None declared

Ethical approval: Not required

\section{REFERENCES}

1. Zimmermann EM, Christman GM. Approach to the female patient with gastrointestinal disease. In: $\mathrm{T}$ Yamada, DH Alpers, C Owyang, DW Powell, FE Silverstein, eds. Textbook of Gastroenterology. Philedelphia, Pa, USA: Lippincott. 1995:1023-43.

2. Buanga KJ, Alcazar JL, Laparte MC. Catamenial rectal bleeding and sigmoid endometriosis. J Gynecol Obstet Biol Reprod (Paris). 1992;21:773-4.

3. Beltran MA, Tapia QTF, Araos HF, Martinez GH, Cruces KS. Ileal endometriosis as a cause of intestinal obstruction: Report of two cases. Rev Med Chil. 2006;134:485-90.

4. Bulun SE. Endometriosis. N Engl J Med. 2009;360:268-79.

5. Bergqvist A. Different types of extragenital endometriosis: a review. Gynecol Endocrinol. 1993;7:207-21.

6. Duepree HJ, Senagore AJ, Delaney CP, Marcello CP, Brady KM, Falcone T. Laparoscopic resection of deep pelvic endometriosis with rectosigmoid Involvement. J Am Coll Surg. 2002;195:754-8.

7. Yantiss RK, Clement PB, Young RH. Endometriosis of the intestinal tract: a study of 44 cases of a disease that may cause diverse challenges in clinical and pathologic evaluation. Am J Surg Pathol. 2001;25:445-54.

8. Varras M, Kostopanagiotou E, Katis K, Farantos CH, Angelidou-Manika Z, Antoniou S. Endometriosis causing extensive intestinal obstruction simulating carcinoma of the sigmoid colon: a case report and review of the literature. Eur $\mathbf{J}$ Gynaecol Oncol. 2002;23:353-7.

9. Won KH. Endometriosis, mucocele, and regional enteritis of Meckel's diverticulum: An unusual case report and experiences in 111 cases. Archives of Surgery. 1969;98(2):209-12.
10. Bergemann W, Heuer C. Extragenital endometriosis with multiple stenoses of the small intestine. Fortschritte der Medizin. 1992;110(15):281-4.

11. Witz CA. Current concepts in the pathogenesis of endometriosis. Clinical Obstetrics and Gynecology. 1999;42(3):566-85.

12. Minh HN, Smadja A, Orcel L. An integrated histogenetic concept of internal and external endometriosis. Journal de Gyn'ecologie, Obst'etrique et Biologie de la Reproduction. 1986;15(1):29-35.

13. Quinn M. Endometriosis: the consequence of neurological dysfunction. Medical Hypotheses. 2004;63(4):602-08.

14. Ouinn MJ. Endometriosis: The consequence of uterinedenervation - reinnervation. Archives of Gynecology and Obstetrics. 2011;284:1423-9.

15. Donnez J, Spada F, Squifflet J, Nisolle M. Bladder endometriosis must be considered as bladder adenomyosis. Fertil Steril. 2000;74:1175-81.

16. Anaf V, Nakadi IE, Simon P. Preferential infiltration of large bowel endometriosis along the nerves of the colon. Human Reproduction. 2004;19(4):996-1002.

17. Langlois NE, Park KG, Keenan RA. Mucosal changes in the large bowel with endometriosis: a possible cause of misdiagnosis of colitis. Hum Path. 1994;25:1030-4.

18. Agic $\mathrm{A}, \mathrm{Xu} \mathrm{H}$, Finas D. Is endometriosis associated with systemic subclinical inflammation?. Gynecol Obstet Invest. 2006;62:139-47.

19. Bazot M, Darai E, Hourani R, Thomassin I, Cortez A, Uzan S, et al. Deep pelvic endometriosis: MR imaging for diagnosis and prediction of extension of disease. Radiology. 2004;232:379-89.

20. Loubeyre $P$, Petignat $P$, Jacob S, Egger JF, Dubuisson JB, Wenger JM. Anatomic distribution of posterior deeply infiltrating endometriosis on MRI after vaginal and rectal gel opacification. AJR Am J Roentgenol. 2009;192:1625-31.

21. Biscaldi E, Ferrero S, Fulcheri E, Ragni N, Remorgida V, Rollandi GA. Multislice CT enteroclysis in the diagnosis of bowel endometriosis.Eur Radiol. 2007;17:211-9.

22. Teke Z, Aytekin FO, Atalay AO, Demirkan NC. Crohn's disease complicated by multiple stenoses and internal fistulas mimicking small bowel endometriosis. World Journal of Gastroenterology. 2008;14(1):146-51.

23. Kavallaris A, Köhler C, Kühne-Heid R, Schneider A. Histopathological extent of rectal invasion by rectovaginal endometriosis. Hum Reprod. 2003;18(6):1323-7.

24. Bedaiwy MA, Falcone T. Laboratory testing for endometriosis.Clin Chim Acta. 2004;340:41-56.

25. Mounsey AL, Wilgus A, Slawson DC. Diagnosis and management of endometriosis. Am Fam Physician. 2006;74:594-600.

26. Ferrero S, Camerini G, Ragni N, Menada MV, Venturini PL, Remorgida V. Triptorelin improves intestinal symptoms among patients with colorectal 
endometriosis. Int J Gynaecol Obstet. 2010;108:2501.

27. Ferrero S, Camerini G, Ragni N, Venturini PL, Biscaldi E, Remorgida V. Norethisterone acetate in the treatment of colorectal endometriosis: a pilot study. Hum Reprod. 2010;25:94-100.

28. Whelton C, Bhowmick A. Acute endometrial bowel obstruction-A rare indication for colonic stenting. Int J Surg Case Rep. 2013;4:160-3.

29. Yap C, Furness S, Farquhar C. Pre and post operative medical therapy for endometriosis surgery. Cochrane Database Syst Rev. 2004;3:CD003678.
30. Biliatis I, Akrivos N, Sotiropoulou M, Rodolakis A, Simou M, Antsaklis A. Endometrial stromal sarcoma arising from endometriosis of the terminal ileum: the role of immunohistochemistry in the differential diagnosis.Journal of Obstetrics and Gynaecology Research. 2012;38(5):899-902.

Cite this article as: Mishra G, Vats M, Pandey D, Akhtar A. Small bowel obstruction due to subserosal endometriosis: an elusive condition. Int J Reprod Contracept Obstet Gynecol 2016;5:907-12. 